\title{
LA ADICCIONOLOGÍA UNA ESPECIALIDAD MÉDICA Y SUBESPECIALIDAD DE LA PSIQUIATRÍA
}

\author{
ADDICTIONOLOGY A MEDICAL SPECIALTY AND SUB-SPECIALTY OF PSYCHIATRY \\ Martín Nizama-Valladolid ${ }^{1, a, b}$, Fernando Luna ${ }^{1, a, b}$, Carlos Cachay ${ }^{1, a, b}$
}

\begin{abstract}
RESUMEN
La Adiccionología como componente de las ciencias de la salud, está alcanzando un rápido reconocimiento como disciplina médica, específicamente como subespecialidad de la psiquiatría. El problema de las adicciones es un tema que se extiende y se agrava en la sociedad posmoderna, debido a su multidimensionalidad y multifactorialidad. Las condiciones de salud de los países han mejorado en cuanto a la reducción de la mortalidad y de la incidencia de algunas enfermedades transmisibles. Sin embargo, se está dando una mayor incidencia de enfermedades crónicas no transmisibles relacionadas con los estilos de vida, así como la emergencia de problemas de salud derivados de la violencia, el consumo de drogas, adicciones sociales, lúdicas y adicción digital (conectividad). Los factores familiares, incluyendo el rechazo de los padres y los conflictos familiares, debido al abuso de sustancias y problemas de salud mental, se encuentran entre los predictores más fuertes y consistentes de los problemas de comportamiento de los adolescentes. De otro lado, en el Modelo Holístico Centrado en la Familia, Nizama propone el desarrollo humano integral mediante la organización multidimensional como eje de vida que comprende once dimensiones de la vida humana: corporal, potencial genético, personalidad, esencia humana, cognición, imaginación, estilos de vida, activos, imagen, futuridad y posteridad. En conclusión, la adiccionología es la especialidad médica indicada para el manejo exitoso y esperanzador de esta enfermedad volitiva en todos los grupos de edad, con adherencia familiar comprometida y sostenible.
\end{abstract}

Palabras clave: Adicción; Psiquiatría; Medicina de las adicciones; Familia. (fuente: DeCS BIREME)

\begin{abstract}
Addictionology as a component of the health sciences is rapidly becoming recognized as a medical discipline, specifically as a subspecialty of psychiatry. The problem of addictions is a subject that extends and worsens in postmodern society, due to its multidimensionality and multifactoriality. The health conditions of the countries have improved in terms of reducing mortality and the incidence of some communicable diseases. However, there is a greater incidence of chronic noncommunicable diseases related to lifestyles, as well as the emergence of health problems derived from violence, drug consumption, social, recreational addictions and digital addiction (connectivity). Family factors, including rejection by parents and family conflicts, due to substance abuse and mental health problems, are among the strongest and most consistent predictors of adolescent behavior problems. Therefore, in the Holistic Family Centered Model, Nizama proposes integral human development through multidimensional organization as an axis of life that comprises eleven dimensions of human life: body, genetic potential, personality, human essence, cognition, imagination, lifestyles, assets, image, futurity and posterity. In conclusion, addictionology is the medical specialty indicated for the successful and hopeful management of this volitional disease in all age groups, with committed and sustainable family adherence.
\end{abstract}

Key words: Addiction; Psychiatry; Addiction medicine; Family. (source: MeSH NLM)

${ }^{1}$ Departamento Académico de Psiquiatría, Facultad de Medicina, Universidad Nacional Mayor de San Marcos, Lima-Perú.

a Médico psiquiatra.

${ }^{\mathrm{b}}$ Doctor en Medicina.

Citar como: Martín Nizama-Valladolid, Fernando Luna, Carlos Cachay. La adiccionología una especialidad médica y subespecialidad de la psiquiatría. [Artículo de Revisión].2019;19(2):00-00. (Abril 2019). DOI 10.25176/RFMH.v19.n2.2078 


\section{INTRODUCCIÓN}

Desde la perspectiva integral de las ciencias de la salud basada en evidencias en el campo de la genética y el funcionamiento cerebral, se posiciona a las adicciones como una especialidad médica y sub-especialidad de la psiquiatría. Con este objetivo se aborda la complejidad multicausal y multidimensional de esta entidad nosológica; así como los modelos y teorías modernas que son los paradigmas de esta área de la medicina. Por ello se revisa, analiza e integra los diferentes enfoques predominantes; desde la biogenética y los estilos de vida hasta los constructos familiares y comunitarios; todo lo cual ha posibilitado aplicar en el ámbito de los sistemas prestacionales de salud, los principios para desarrollar e implementar programas terapéuticos exitosos a largo plazo, tras varios años de intervención sistemática ${ }^{10}$.

Igualmente, se analiza el impacto de las actuales transformaciones demográficas y epidemiológicas causados por la globalización, que han determinado cambios críticos estructurales en la población y en las organizaciones sociales, especialmente en la familia natural, otrora célula básica de la sociedad y en la actualidad desplazada por el individualismo extremo, en el contexto de una sociedad posmoderna esencialmente materialista, pragmática, hedonista, banal y deshumanizada, dominada por el conocimiento, la informática, la tecnología, el confort y el consumismo impuesto por el mercado. En este mundo globalizado, la moda es que la familia natural ha sido convertida en un ente meramente procreador y proveedor, privada de autoridad, de rol educador y de ser el eje social de la esencia humana. Como tal, la familia ha dejado de ser fuente de educación: amor, principios rectores, valores de vida y sentimientos superiores.

Este contexto ha sido propicio para que en el área de las adicciones en el s. XXI se afronten nuevos retos como:

1. Surgimiento de nuevas drogas de síntesis; además de las drogas clásicas que en la actualidad son manipuladas genéticamente y otras adicciones relacionadas a la tecnología digital como videojuegos, redes sociales, cibernavegación y ludopatía cibernética.

2. Cambios sustantivos en el perfil demográfico y epidemiológico de las adicciones; puesto que se ha pasadodeunapatologíaqueerapredominantemente de varones y adultos monoconsumidores a una enfermedad en la cual cada día se incrementan las usuarias mujeres; al igual que los niños, adolescentes y jóvenes de ambos géneros. En la actualidad prevalece el policonsumo, la poliadicción y la adicción familiar mútiple en el hogar; por ejemplo alcoholismo y tabaquismo, dependencia a psicofármacos y adicción a sustancias psicoactivas ilícitas; así como una elevada prevalencia de comorbilidad psiquiátrica que excede del 70 por ciento.

3. Los cuatro tipos de adicciones existentes: a. Sociales, b. Lúdicas, c. Digitales y d. Químicas, tienen como núcleo único el circuito mesolímbico cortical, centro del reforzamiento positivo y negativo, lo cual hace de las adicciones una enfermedad única no sujeta a fragmentación.

4. Anomia familiar patológica. Caos estructural y funcional familiar en cuyo seno se incuban y se sostienen las diversas adicciones'.

El deterioro psicoorgánico causado por las diferentes adicciones es la base neurobiológica de los síndromes clínicos de esta entidad mórbida, cuyas principales manifestaciones son la inconsciencia de enfermedad, la pérdida de la voluntad, la pérdida del control de impulsos e insensibilidad. Comprende la neurodegeneración y la disrupción de los circuitos neuronales del lóbulo prefrontal del cerebro ${ }^{10,11,12,13}$. Por ello, la DEIDAE de Adicciones del Instituto Nacional de Salud Mental "Honorio Delgado-Hideyo Noguchi", desde su creación hace 24 años ha aplicado modelos terapéuticos alternativos con programas integrales en el abordaje de la enfermedad adictiva, con enfoque de género y dirigido a poblaciones vulnerables: niños, adolescencia y jóvenes.

Es importante el conocimiento científico profundo de las adicciones para saber cómo impactan en la sociedad, la familia y las personas adictas, a fin de prevenir esta pandemia mediante la promoción de estilos de vida saludables y una cultura de valores. Igualmente, mejorar la calidad de vida de las personas afectadas por esta enfermedad volitiva, promover atenciones de calidad a cargo de equipos científicamente competentes y aptos; así como una percepción objetiva, no estigmatizada de la enfermedad adictiva. Así mismo, debe superarse el intrusismo por parte de profesionales inaptos que creyendo saber manejar una enfermedad extremadamente compleja que en realidad desconocen en su esencia clínica y complejidad familiar y social, exponen a los pacientes al síndrome de la "puerta giratoria", a la creencia de la incurabilidad de las adicciones, al probable desenlace funesto ${ }^{8,9}$ y a la desesperanza familiar y colectiva. 
El conocimiento de las adicciones ha tenido cambios acelerados e importantes en las últimas décadas, como resultado de los avances de las investigaciones recientes sobre el funcionamiento de los genes y del cerebro humano; así como en los estudios sobre la familia y los grupos humanos en el campo psicosocial, lo cual ha viabilizado la construcción de un marco teórico de las adicciones como disciplina de las ciencias de la salud y de la conducta humana, que ha conducido a diseñar e implementar modelos de intervención con programas terapéuticos exitosos a largo plazo, aplicados por equipos interdisciplinarios especializados, con las competencias necesarias para el abordaje de la enfermedad adictiva, involucramiento y adherencia plena de la familia comprometida en el proceso terapéutico integral.

La Adiccionología como componente de las ciencias de la salud, está alcanzando un rápido reconocimiento como disciplina médica, específicamente como subespecialidad de la psiquiatría, lo cual en gran medida se debe a los grandes avances científicos, particularmente en el ámbito de la genética con el proyecto del genoma humano, el mapeo cerebral mediante el proyecto "Brain Research through Advancing Innovative Neurotechnologies (BRAIN), Working Group Report to the Advisory Committee to the Director, NIH" y del conectoma humano. La consolidación de los nuevos conocimientos en este campo de la medicina relacionados con las adicciones, posibilita una percepción clara y objetiva de este fenómeno mórbido extremadamente complejo por su multidimensionalidad y multifactorialidad, que conduce a ser abordado como las dos caras de una misma moneda: 1. Como problema de salud pública y 2. Como enfermedad, una subespecialidad de la psiquiatría denominada Adiccionología.

A simismo, la Adiccionología como rama de la medicina, tanto en su epistemología como en su hermenéutica y nosología, está construida sobre marcos teóricos y modelos científicos aceptados internacionalmente como la teoría general de sistemas, teorías del desarrollo de la personalidad, teorías del aprendizaje, teoría de los grupos humanos, las neurobiología, las neurociencias, la genética y la sociobiología, entre otros marcos conceptuales que fundamentan los modelos que son paradigmas en el estudio y abordaje de la conducta humana , como el Modelo Holístico Centrado en la Familia, con sus dimensiones ecológicas, biológicas, psíquicas, familiares, sociales y espirituales, y el "Modelo Biopsicosocial para el afronte de pacientes (Anales de Salud Mental 1991 VOL VII) .
Tras la globalización y el advenimiento del siglo XXI, con el desarrollo tecnológico, la deshumanización social masiva y el desplazamiento de la familia por el individualismo extremo, las adicciones se convirtieron en un problema de salud pública. La aparición de nuevas sustancias psicoactivas elaboradas sintéticamente, las drogas clásicas manipuladas genéticamente y las nuevas adicciones a la droga digital (conectividad), producto de la revolución en la comunicación tecnológica con la Internet, ha propiciado la aparición de nuevas formas de patología adictiva como la adicción digital (videojuegos, redes sociales y cibernavegación), que afectó a poblaciones vulnerables como son las mujeres y grupos etarios específicos como la niñez, la adolescencia y la juventud. Esta situación se ha constituido en un verdadero reto para los profesionales especializados en el afronte de estas nuevas formas de adicción y los ha obligado a que desarrollen programas integrales orientados a estas poblaciones, con necesidades urgentes de tratamiento especializado y efectivo a largo plazo.

\section{LA ADICCIONOLOGÍA: ESPECIALIDAD MÉDICA}

Las drogas están presentes en todas las sociedades, constituyendo el centro de una dinámica social, económica, ética y política. Desde la antigüedad las sustancias psicoactivas han formado parte del pensamiento religioso y científico, aunque también han servido para justificar guerras y diversos procesos de exclusión social, conflictos geopolíticos de los cuales estas sustancias suelen ser el móvil oculto como el narcotráfico y el narcoterrorismo en el VRAEM (Valle de los ríos Apurímac, Ene y Mantaro) peruano. En la actualidad el problema de las adicciones es un tema de mercado que se extiende y se agrava en la sociedad posmoderna. Los Estados nacionales han comenzado a sensibilizarse al problema de las adicciones, en el cual las complicidades y la corrupción juegan un rol determinante para degradar la conciencia ética de las instituciones policiales, judiciales, políticas y sociales, para de esta manera lograr la permisividad y el consentimiento de sus actividades en los diferentes niveles y así poder operar con impunidad e incluso impulsar la legalización de drogas como la marihuana y la cocaína, con grave daño a la salud individual, familiar y colectiva.

Un fenómeno tan complejo como las adicciones, debido a su multidimensionalidad y multifactorialidad, ha requerido de la estructuración de un riguroso cuerpo de conocimiento científico, basado en evidencias logradas en las últimas décadas con el progreso de las ciencias médicas y sociales. Todo 
este conocimiento ha permitido que se desarrollen programas terapéuticos basados en evidencias científicas, los cuales con la intervención de equipos profesionales interdisciplinarios especializados en Adiccionología, han permitido que sean catalogados como exitosos, logrando indicadores de adherencia, disminución de recaídas y logros de reinserción social equiparados o que superan a los logrados en los tratamiento de enfermedades crónicas como las oncológicas, las metabólicas (diabetes Mellitus), las cardiovasculares (Hipertensión Arterial) y HIV/SIDA, entre otras.

Esta subespecialidad de la psiquiatría ha sido reconocida académicamente en el ámbito internacional y nacional. Es así que la Universidad Nacional Mayor de San Marcos, desde el año 2011 inició la formación de médicos psiquiatras en esta subespecialidad médica.

La Adiccionología se basa en los siguientes principios:

1. La adicción es una enfermedad crónica que afecta a todas las etapas de la vida y no únicamente a la adolescencia. Si bien tiene una alta prevalencia durante esta etapa de la vida, es una enfermedad compleja por su multicausalidad y elevada tasa de comorbilidad. Sin intervención médica especializada la historia natural puede tener un desenlace tanático. El abordaje de esta enfermedad es de amplio espectro. Comprende aspectos de políticas públicas de salud que modulen y articulen la intra e intersectorialidad con la intervención de equipos interdisciplinarios especializados en Adiccionología.

2. Actualmente en el ámbito internacional se reconoce la especialidad de Adiccionología, por tanto el tratamiento en esta área, más aún en las instituciones de salud con cobertura de atención de alta complejidad, es competencia de los profesionales de salud especializados en esta patología volitiva.

3. El perfil del médico Adiccionólogo es el de un especialista con las siguientes competencias.

- Poseer conocimientos sólidos y suficientes de los factores biológicos, psíquicos y socioculturales que le permitan comprender a cabalidad el fenómeno adictivo en su integridad y variabilidad, como son las adicciones químicas, adicción digital, ludopatía y adicciones sociales.

- Aplicar conocimientos actuales, investigar e innovar estrategias preventivas promocionales para liderar y asesorar las políticas públicas orientadas a detener y reducir la incidencia.
- Aplicar los conocimientos actuales, innovar e investigar estrategias diagnósticas y terapéuticas en individuos con adicción en los diversos grupos etarios y de género.

- Conocer a profundidad la farmacocinética, farmacodinamia, reacciones adversas y toxicología de las drogas de abuso en el ser humano (con sus variantes según género y grupo etario), para manejar con idoneidad los fenómenos propios de la patología adictiva: intoxicación aguda y crónica, síndrome de abstinencia, craving, consecuencias a largo plazo del uso de las mismas, entre otros.

- Manejar las nuevas adicciones como la adicción digital (conectividad), ludopatía, adicciones sociales y otras conductas adictivas atípicas, dominando el conocimiento de la base neurobiológica común a los diferentes tipos de adicciones; al igual que las consecuencias biológicas y el deterioro en el ámbito personal, familiar, académico, laboral y social.

- Manejar la multidimensionalidad de la patología adictiva en sus componentes psicofisiológico, orgánico, psíquico, comorbilidad y anomia familiar patológica; así como la desintoxicación, deshabituación y rehabilitación, cuyo manejo con experticia posibilita la curación y el desarrollo humano integral, individual y familiar.

4. Como toda enfermedad crónica, el tratamiento de la patología adictiva es un proceso a largo plazo que comprende 3 a 5 años o más, lo cual rebasa el criterio de edad. Durante el prolongado proceso de tratamiento, rehabilitación, curación y desarrollo humano integral, se presentan transiciones del ciclo vital; igualmente puede haber recaídas, eventos de comorbilidad que requieren ajustes continuos en el manejo farmacológico y psicoterapéutico, individual y familiar. Es más, el tratamiento necesariamente está centrado en revertir la anomia familiar patológica que incuba y sostiene las adicciones.

\section{PRINCIPIOS PARA EL TRATAMIENTO DE LAS ADICCIONES ${ }^{14}$}

Según el Instituto Nacional de Drogas y Alcohol de los Estados Unidos (NIDA), los principios recomendados para el tratamiento son los siguientes:

1. No existe un tratamiento único que sea apropiado para todos los individuos.

2. Los tratamientos efectivos deben estar disponibles de inmediato, cuando el cliente los necesite.

3. Los tratamientos efectivos atienden múltiples necesidades del individuo y no sólo el uso de drogas. 
4. Tanto el tratamiento como el plan de servicios que se ofrece a un individuo debe ser evaluado y modificado constantemente para asegurar que atiende las necesidades del cliente.

5. Permanecer en un tratamiento por un período adecuado de tiempo (3 a 6 meses mínimo para la primera fase) es crítico para la efectividad del mismo.

6. La consejería individual y de grupo, así como otras terapias conductuales, son componentes esenciales para la efectividad de un tratamiento de adicciones.

7. La administración de medicamentos (farmacoterapia) es un elemento importante en el tratamiento de algunos pacientes, especialmente cuando se combinan con consejería y otras terapias conductuales.

8. Personas en uso, abuso o dependencia de drogas que presentan además otro trastorno mental (doble diagnóstico) tienen que recibir tratamiento para ambos trastornos en una forma integrada.

9. La desintoxicación médica es sólo la primera etapa del tratamiento de adicciones y por sí sola hace muy poco para cambiar el uso o abuso de drogas prolongado (a largo término)

10. El tratamiento no necesita ser voluntario para ser efectivo.

11. La posibilidad de uso de drogas ("lapsos") durante el tratamiento debe ser supervisado constantemente.

12. Los programas de tratamiento deben proveer evaluación para VIH/SIDA, Hepatitis B y C, tuberculosis y otras enfermedades infecciosas y deben proveer también consejería sobre conductas de riesgo y prevención de dichas enfermedades.

13. La recuperación de la dependencia de drogas puede ser un proceso a largo término $y$ frecuentemente requiere de múltiples episodios de tratamiento.

En nuestro medio la Dirección Ejecutiva de Investigación, Docencia y Atención Especializada de Adicciones del Instituto Nacional de Salud Mental "Honorio Delgado-Hideyo Noguchi" (INSM "HD-HN"), aplica los siguientes principios terapéuticos:

1. No existe un tratamiento único aplicable a todos los casos. El abordaje es multimodal y a largo plazo.
2. Los tratamientos efectivos deben ser accesibles de inmediato cuando el paciente los requiera.

3. Los tratamientos efectivos atienden múltiples necesidades del individuo y el entorno familiar, y no únicamente la conducta adictiva.

4. El plan de trabajo y el tratamiento que se ofrecen al paciente debe ser evaluado y reajustado continuamente, para asegurar que se atiende las necesidades clínicas del paciente y de su entorno familiar.

5. Permanecer en un programa terapéutico formulado científicamente por un período de tiempo de 3 a 5 años, puede asegurar el desarrollo humano integral y con ello la curación de la adicción.

6. La psicoterapia individual y familiar son componentes esenciales para la efectividad del tratamiento de las adicciones.

7. La farmacoterapia permite el manejo del craving, síndrome de abstinencia, excitación psicomotora, conducta disocial y flashback, entre otras manifestaciones clínicas de las adicciones.

8. Personas adictas con comorbilidad requieren tratamiento específico para los diferentes trastornos operados de manera integrada.

9. La desintoxicación y la deshabituación es sólo el primer paso del tratamiento integral de las adicciones.

10. En el abordaje terapéutico de las adicciones se incluye la anomia familiar patológica, la poliadicción y la adicción familiar múltiple.

11. El abordaje terapéutico se basa en la clínica de las adicciones y no en ideologías dogmáticas, prejuicios cientificistas ni está sujeto a conflicto de intereses ajenos a la Adiccionología.

12. El tratamiento no necesita ser voluntario para ser efectivo. El tratamiento involuntario es frecuente y lo decide la familia o un juez. La intervención involuntaria impide la degradación y la decrepitud del enfermo; así como la desintegración familiar, abandono de sí mismo, prisión u occisión del adicto $^{62}$

13. La recaída es común en la adicción y no debe ser causa de descontinuación del tratamiento ni motivo de deserción. Esta posibilidad debe ser considerada constantemente durante todo el tratamiento. 
14. Los programas de tratamiento deben proveer evaluación para VIH/SIDA, Hepatitis B y C, tuberculosis y otras enfermedades infecciosas. También deben brindar consejería sobre conductas de riesgo y prevención de dichas enfermedades.

15. El tratamiento de las adicciones es a largo plazo y frecuentemente requiere de varios episodios de intervención.

16. En la Dirección de Adicciones en la actualidad se aplican tres modelos de intervención interdisciplinaria con participación activa de la familia, mediante la ejecución de tres programas:

\section{Modelo Holístico Centrado en la Familia}

\section{Biopsicosocial}

Integrativo

Los resultados más óptimos en el proceso de curación de los pacientes han sido observados tras la fidelización de la persona y de su familia al tratamiento. Para lograrlo son dos los factores considerados:

1. La adherencia o integración. Se refiere al número de exposiciones del paciente a sesiones y a la aplicación de un número de procedimientos establecidos en los programas de cada uno de los tres modelos de intervención.

2. La competencia que comprende las habilidades y experticia del equipo terapéutico para aplicar el modelo adoptado, el tiempo previsto para las intervenciones y el grado de respuesta de las conductas de los pacientes; lo cual es entendido en el contexto de la relación terapéutica ${ }^{10}$.

Es decir para que ambos procesos se den se requiere de la existencia previa de modelos con sus respectivos programas terapéuticos; pues, sin ellos no se puede hablar de fidelización, o sea, ni de adherencia ni de competencia. En este sentido es una fortaleza que en la Dirección de Adicciones del Instituto, existan tres programas familiares de intervención sustentados en modelos y marcos conceptuales científicos vigentes en el ámbito internacional.

\section{NUEVOS RETOS: ADICCIONES EN POBLACIONES VULNERABLES: NIÑOS, ADOLESCENTE Y JÓVENES.}

Con el advenimiento del siglo XXI signado por la globalización, se produjeron prodigiosos progresos tecnológicos que acarrearon enormes cambios cuantitativos y cualitativos que modificaron radicalmente la conducta humana, la vida en sociedad, los estilos de vida y el sistema ecológico, con la consecuente crisis de cultura y civilización que interpela la conciencia del mundo cuestionando los valores y principios que sustentan las sociedades. La salud se constituye en un punto de encuentro, donde confluyen modelos teóricos de los componentes ecológico, biológico, psíquico y social; así como el individuo, la familia y la comunidad; igualmente concurren los componentes social, económico y ético. La salud, además de su valor intrínseco, se ha constituido en un medio para la realización personal y colectiva de la sociedad. Sobre estas realidades las adicciones se incrementan en las poblaciones, agravándose con la aparición de nuevas sustancias y comportamientos adictivos como la ludopatía y la adicción digital que impactan especialmente a la población adolescente y joven de la sociedad posmoderna. Ellos, al iniciar cada vez más precozmente el uso de drogas químicas, sociales y digitales se convierten en futuros ciudadanos de los países (bono demográfico) afectados en su salud. Entonces, dejan de ser un producto social positivo necesario con generaciones de individuos saludables que aseguren su participación dinámica y activa en la construcción y desarrollo de una sociedad de bienestar y de bien-ser.

Las condiciones de salud de los países han mejorado en cuanto a la reducción de la mortalidad y de la incidencia de algunas enfermedades transmisibles. Sin embargo, se está dando una mayor incidencia de enfermedades crónicas no transmisibles relacionadas con los estilos de vida, así como la emergencia de problemas de salud derivados de la violencia, el consumo de drogas ${ }^{14}$, adicciones sociales, lúdicas y adicción digital (conectividad).

Paralelamente, los cambios en la pirámide poblacional y la transición epidemiológica ha dado como resultado que en los países de ingresos medios como el Perú se encuentren estratos socio-demográficos con enfermedades propias de países de ingresos altos como enfermedades crónicas no trasmisibles: diabetes Mellitus e HPTA, entre otras, junto con enfermedades infecto-contagiosas propias de sociedades de bajos ingresos como TBC, dengue, chikungunya. Ante este cambio en el perfil demográficoy epidemiológico delas enfermedades, las evidencias también indican que en el área de las adicciones se ha pasado rápidamente de una patología que era predominantemente de varones y adultos monoconsumidores, a una enfermedad en la que cada día se incrementan las usuarias mujeres; así como los niños, adolescentes y jóvenes de ambos géneros, comúnmente policonsumidores y 
poliadictos. Igualmente, se incrementa el porcentaje de adicción familiar múltiple. Para responder a este desafío la Dirección de Adicciones del Instituto, desde hace 24 años desarrolla tres modelos terapéuticos con programas integrales para el abordaje de la enfermedad adictiva con enfoque de género, dirigido a poblaciones vulnerables: niños, adolescentes y jóvenes.

El abuso de sustancias entre los adolescentes rara vez se produce en forma aislada de otros trastornos del desarrollo y desórdenes psiquiátricos ${ }^{15}$. Los estudios con muestras clínicas y de casos judiciales de menores en la comunidad, documentan al igual que el abuso de sustancias en adolescentes, que frecuentemente es comórbido con múltiples trastornos psiquiátricos, principalmente trastorno por déficit de atención e hiperactividad (TDAH), depresión y trastorno por estrés postraumático (TEPT) $16,17,18,19,20$. En adolescentes, las tasas de comorbilidad del uso de sustancias con trastornos psiquiátricos son en promedio el $60 \%$ en las muestras de la comunidad [6] y pueden incrementarse hasta el 80-90 \% en muestras del Departamento de Justicia de Menores en los Estados Unidos 21,22,23,24 Varios estudios demuestran que el compromiso con el tratamiento y los resultados exitosos de los mismos, son más difíciles de lograr en adolescentes con patología co-mórbida $23,25,26,27$.

El problema en adolescentes mayormente es externalizante (se refieren a las alteraciones del control de los comportamientos tales como agresión, impulsividad, negativismo desafiante, hiperactividad y problemas de conducta disocial, Achenbach \& Edelbrock, 1983; Roca \&Alemán, 2000). Porello, muchos jóvenes abusadores de sustancias con trastornos de conducta graves están en riesgo de progresar a un trastorno disocial de la personalidad, experimentar abuso crónico de sustancias, tener problemas de salud mental, el empleo, la salud y dificultades de relación en la edad adulta ${ }^{28,29}$. Los modelos "factor común" y la "desviación en general", a menudo son invocados para explicar la estrecha asociación entre el consumo de sustancias y los trastornos psiquiátricos, principalmente problemas de externalización, como el desorden de conducta ${ }^{23}$. De hecho, como postula Jessor y Jessor ${ }^{37}$ en la "teoría de la conducta problema," el abuso de sustancias en adolescentes tiende a concurrir con una variedad de otras conductas disruptivas que pueden tener consecuencias a largo plazo, incluidas las prácticas sexuales de riesgo, el fracaso escolar y social, el aislamiento, la alienación y el conflicto. La agrupación coherente de estas conductas y la evidencia de que pueden tener efectos recíprocos ${ }^{31}$ ha conducido al examen de los factores de riesgo comunes que pueden explicar una serie de problemas de la adolescencia, como el temperamento temprano y vulnerabilidades ambientales ${ }^{32,33}$. Dado que se conocen factores de riesgo comunes para contribuir al abuso de sustancias y otros trastornos en la adolescencia, frente a estas vulnerabilidades y la promoción de procesos de protección a través de la intervención específica, puede tener efectos amplios y duraderos ${ }^{34,35}$.

Los factores familiares, incluyendo el rechazo de los padres y los conflictos familiares, la supervisión ineficaz y estrategias de disciplina de los progenitores, y el funcionamiento de los padres en peligro, debido al abuso de sustancias y problemas de salud mental, se encuentran entre los predictores más fuertes y consistentes de los problemas de comportamiento de los adolescentes, tales como el abuso de sustancias y trastornos de conducta ${ }^{36,37}$. Los factores protectores dentro de la familia también pueden amortiguar el impacto negativo de los factores de riesgo, tales como el involucramiento con pares desviados ${ }^{38}$. En un estudio reciente, se observó que el apoyo e involucramiento en la crianza de los hijos presenciales disminuye el riesgo genético para el abuso de drogas en adolescentes ${ }^{46}$. Las asociaciones consistentes entre los factores familiares y los problemas de comportamiento de los adolescentes, así como un fuerte apoyo empírico para las intervenciones basadas en la familia a prueba en una serie de ensayos rigurosamente controlados en las últimas dos décadas ${ }^{38,39}$, ha establecido firmemente la participación de la familia como un ingrediente crítico en el tratamiento de los trastornos por consumo de sustancias en adolescentes ${ }^{40} . \mathrm{Y}$, debido a que los factores familiares predicen una serie de problemas en la adolescencia, incluyendo la angustia internalizada y las conductas de externalización, intervenir para cambiar las interacciones negativas y patrones dentro de la familia, también puede ser crítico al impactar tanto en los trastornos concurrentes como en el abuso de sustancias durante la adolescencia.

Desafortunadamente, mientrasquevariostratamientos han demostrado eficiencia en reducir el uso de sustancias de los adolescentes ${ }^{38,39}$, muchas de estas intervenciones se centran en aliviar el uso específico de las sustancias, en lugar de la focalización de múltiples problemas de comportamiento de los adolescentes. La investigación clínica que excluye deliberadamente casos complejos debido a múltiples comorbilidades no ha contribuido a esclarecer lo complejo y heterogéneo 
de la nosología de la enfermedad adictiva. Así, hay escasos ejemplos de intervenciones de amplia base que abordan simultáneamente y con eficacia el abuso de sustancias, conductas de riesgo relacionadas, tales como las prácticas sexuales inseguras, la delincuencia, los síntomas psiquiátricos y problemas escolares entre los adolescentes ${ }^{23}$. Considerando que el abuso de sustancias entre los adolescentes tiende a co-ocurrir y puede exacerbar (y exacerbarse por) otros problemas emocionales y de comportamiento, las intervenciones para esta población deben abordar múltiples comorbilidades $^{40}$. De hecho, las intervenciones integrales que tratan simultáneamente la comorbilidad son las recomendadas con frecuencia ${ }^{19,20,41}$, pero son escasos en la práctica.

En los últimos documentos de orientación y revisión de trastornos comórbidos, tanto para los adolescentes y adultos, varios expertos indican intervenciones para el abuso de sustancias y problemas de salud mental que se entregan de una manera verdaderamente integrada en lugar de tratamientos distintos ofrecidos simultáneamente o consecutivamente ${ }^{20,41}$.

Respecto al género es importante considerar no sólo el tratamiento según género, sino el tratamiento de subgrupos específicos de mujeres con adiciones que incluyan a las gestantes con Trastorno de Estrés Post-Traumático( TEPT), que sufrieron abuso sexual, Trastorno Alimentario Compulsivo y Trastorno Bipolar, entre otras entidades comórbidas frecuentes. A este subgrupo, no sólo hay que ofrecerles alternativas y programas terapéuticos para las adicciones, sino que a la vez hay que brindarles las condiciones de seguridad asociadas al control clínicamente significativo del trastorno comórbido; por ejemplo reducción de los síntomas del TEPT ${ }^{17}$. Los hallazgos muestran que la aplicación de tratamiento centrado en la patología dual conjuntamente con el tratamiento habitual en programas de tratamiento de abuso de sustancias reduce los síntomas de la enfermedad co-mórbida, y a la vez ayuda a mantener la reducción del consumo. De otro lado, también es necesario incluir y considerar estrategias de tratamiento considerando las diferencias de género, aplicando intervenciones específicas para la reducción de riesgo de $\mathrm{VIH}^{42}$.

La DEIDAE de Adiciones del INSM "HD-HN" ha aplicado estas variables en el ejercicio de la subespecialidad de la Adiccionología, por lo que desde el 1 de abril del 2001 atiende e interna adictos adolescentes, además de otros grupos de edad. Así mismo, desde su creación esta DEIDAE es el único Servicio del país que hospitaliza pacientes mujeres, incluidas adolescentes de este género. Así, los tres programas de intervención de la DEIDAE de Adicciones del Instituto ofertan una atención integral, altamente especializada, no sesgada por variables etarias ni de género. Prueba de ello son las tres organizaciones familiares que suman más de 300 familias comprometidas de manera militante con el tratamiento a largo plazo de pacientes monoadictos y poliadictos, comórbidos o no, sean varones, mujeres, niños, adolescentes $\mathrm{o}$ adultos mayores.

\section{La adicción es una enfermedad, con base neurobiológica.}

La adicción, es una enfermedad psiquiátrica cuya incubación sería el resultado de al menos tres pasos secuenciales que interactúan entre sí inevitablemente con dos factores que son la Vulnerabilidad individual y el Grado/cantidad de exposición a la droga o conducta adictiva. El primer paso, uso esporádico o recreacional es un proceso de aprendizaje mediado por la sobreactivación de los sustratos neurobiológicos del sistema de recompensa natural, que permite que la mayoría de las personas perciban las adicciones como estímulos altamente gratificantes. El segundo paso, uso intensificado, sostenido y en escalada; en el cual la cascada secuencial se produce en algunos individuos vulnerables que poseen un sistema dopaminérgico hiperactivado y una alteración en la función de la corteza prefrontal. El uso sostenido y prolongado de la adicción induce sensibilización al incentivo y un estado alostático que hace que la práctica de la conducta adictiva sea fuertemente deseada y necesitada. Mientras que en la homeóstasis la continuidad e idoneidad del medio se logra mediante la estabilidad, en la alostasis se logra mediante la inestabilidad y el cambio. Los mecanismos alostáticos son capaces de cambiar dentro de una cierta inestabilidad, a modo de amortiguadores biológicos, para que los sistemas homeostáticos permanezcan estables. Es el logro del equilibrio en relación al cambio constante. La formación de hábitos también puede contribuir a estabilizar el uso sostenible de la adicción.

El tercer paso, la pérdida de control de la práctica adictiva que acaba en la adicción, es debida a una segunda vulnerabilidad fenotípica, desencadenada por la exposición a largo plazo y se caracteriza por la pérdida duradera de la plasticidad sináptica en áreas de recompensa en el cerebro que inducen una forma de "cristalización del comportamiento", observada como pérdida de control para la adicción, en donde la ingesta o práctica adictiva ya no es sólo deseada y necesitada, sino también patológicamente sufrida ante su ausencia (síndrome de abstinencia) ${ }^{43}$. 
La adicción como tal, es una enfermedad crónica recidivante caracterizada por la compulsión a buscar, ingerir o practicar la adicción, la pérdida de control para limitar la ingesta o incapacidad de saciamiento y el surgimiento de un estado emocional negativo como disforia, ansiedad e irritabilidad que refleja un síndrome de abstinencia, cuando se impide el acceso a la droga o a la conducta adictiva.

La adicción ha sido conceptualizada como un trastorno volitivo que involucra la impulsividad y compulsividad que producen un ciclo adictivo compuesto por tres fenómenos clínicos:intoxicación impulsiva, abstinencia acompañada de la experiencia afectiva negativa y vivencia del craving: preocupación/anticipación. Estudios de imágenes revelan los circuitos que median los tres estados del ciclo adictivo. Así, para la etapa de intoxicación impulsiva, los elementos clave estarían en las área tegmental ventral y el estriado ventral; para la abstinencia y su experiencia afectiva negativa, se hallaría en la amígdala extendida; y para el craving - la etapa de preocupación/anticipación, estarían implicadas una amplia red que involucra la corteza órbitofrontal, el estriado dorsal, la corteza prefrontal, la amígdala basolateral, el hipocampo y la ínsula; mientras que para la disrupción del control inhibitorio se observa compromiso del gyrus cingular, el gyrus prefrontal dorsolateral y la corteza frontal inferior.

La transición a la adicción implica la neuroplasticidad en todas estas estructuras que pueden comenzar con cambios en el sistema dopaminérgico mesolímbico y una cascada de neuroadaptaciones desde el cuerpo estriado ventral al estriato dorsal y corteza órbitofrontal $y$, eventualmente la disregulación de la corteza prefrontal, la circunvolución cingulada y la amígdala extendida.

La delimitación de los neurocircuitos envueltos en las etapas evolutivas de las formas del síndrome de adicción, constituye una base heurística para la investigación de la neuroadaptación molecular, genética y neurofarmacológica que son claves en el entendimiento de la vulnerabilidad para desarrollar y sostener la adicción. La vulnerabilidad al abuso de drogas está relacionada con la búsqueda de recompensa y la impulsividad, dos constructos que tienen su base biológica en la corteza prefrontal $(\mathrm{PFC})^{44}$.

Sobre la corteza prefrontal y la vulnerabilidad para las adicciones se debe entender que hay sistemas de neurotransmisores de monoamina y amino ácidos situados en subregiones anatómicamente diferentes: la corteza prefrontal medial (mPFC); corteza prefrontal lateral (LPFC); corteza cingulada anterior (ACC) y la corteza órbitofrontal (OFC). Mientras que hay interconexiones complejas y superposición de funciones entre éstas regiones, se piensa que cada una está implicada en diversas funciones relacionadas con las conductas de riesgo para la salud y la vulnerabilidad para el abuso.

Entre las diversas funciones implicadas, la evidencia actual sugiere que la mPFC está involucrada en el procesamiento de la recompensa, atención y restablecimiento del uso de drogas; la LPFC está involucrada en la toma de decisiones, inhibición conductual y es la puerta para la atención; la ACC está involucrada en la atención, el procesamiento emocional y automonitoreo; y la OFC está implicada en la inhibición conductual, la fijación de resultados esperados y sensibilidad a la recompensa/castigo. Las diferencias individuales, por ejemplo edad y sexo influyen en el funcionamiento de estas regiones, las que a su vez impactan en la vulnerabilidad ${ }^{45}$.

El conocimiento adecuado de todas estas variables garantiza el desarrollo de estrategias de prevención y tratamiento para el abuso de sustancias, que deben involucrar los procesos inhibitorios de la PFC, con estrategias y conductas que puedan reducir el riesgo relativo de los comportamientos relacionados al consumo y que incluyan el diseño de servicios públicos efectivos, ejercicios cognitivos, actividad física, entrenamiento de control de retroalimentación y tratamientos farmacológicos ${ }^{45}$.

De otro lado, en el Modelo Holístico Centrado en la Familia, Nizama propone el desarrollo humano integral mediante la organización multidimensional como eje de vida que comprende once dimensiones de la vida humana: corporal, potencial genético, personalidad, esencia humana, cognición, imaginación, estilos de vida, activos, imagen, futuridad y posteridad. Así, la dimensión corporal comprende el aspecto físico de la persona; el potencial genético, las fortalezas y debilidades latentes heredadas de las ramas paterna y materna contendidas en la caja genética o cigoto; la personalidad, el temperamento y carácter que modulan la manera de ser personal; la esencia humana, los principios rectores, los valores de vida y los sentimientos superiores; la cognición, la capacidad de aprender, desaprender, reaprender e innovarse; la imaginación, es la creatividad y el arte de reinventarse, de "morir como soy para renacer como necesito ser"; los estilos de vida, la manera de desenvolverse en la vida cotidiana; los activos, los potenciales genéticos 
convertidos en productos, logros y resultados por acción del quehacer cotidiano; la imagen, la percepción que tienen los otros sobre el actuar de la persona; la futuridad, el mapa mental del devenir que se imagina cada persona; y la posteridad, el legado que aporta el individuo tras dejar de existir. Este Modelo original propone la curación de la adicción y el principal escenario del quehacer terapéutico es la familia y el nido del hogar para el desarrollo humano integral en el lapso de 3 a 5 años, para lograr la felicidad individual y familiar ${ }^{11,46,47}$.

\section{CONCLUSIÓN}

La Adiccionología es la especialidad médica indicada para el manejo exitoso y esperanzador de esta enfermedad volitiva en todos los grupos de edad, con adherencia familiar comprometida y sostenible. La adicción es una enfermedad crónica que requiere tratamiento médico especializado a largo plazo, con el objetivo de lograr la curación con desarrollo humano integral tras varios años de tratamiento familiar e individual, período temporal que excede los límites de los ciclos etarios de la niñez y adolescencia.
Contribuciones de autoría: Los autores participaron en la idea, diseño de proyecto, recolección e interpretación de datos, preparación del manuscrito de la versión final del artículo.

Financiamiento: Autofinanciado.

Conflicto de interés: Los autores declaran no tener conflicto de interés en la publicación de este artículo.

Recibido: 03 de diciembre de 2018

Aprobado: 15 de febrero de 2019

Correspondencia: Martín Nizama-Valladolid

Dirección: Alfredo Salazar 314, Of. 303, Lima 27, Perú

Teléfono: (+511) 421-9939 Anexo 430 y 433 / +51 999-836040

Correo:maniva@terra.com.pe

\section{REFERENCIAS BIBLIOGRÁFICAS}

1. Banco Mundial. "Invertir en Salud". Washington, USA. 1993

2. Bertalanffy, Ludwig Von. "Teoría General de los Sistemas: Fundamentos, desarrollos, aplicaciones" Decimoquinta reimpresión, Editorial Fondo de Cultura Económica. México, 2003

3. Comisión Interamericana Contra el Abuso de Drogas de la Organización de Estados Americanos (CICAD/OEA)- Observatorio Interamericano de Drogas: "Programas para Estimar los Costos Humanos, Sociales y Económicos de las drogas en las Américas- Manual de Investigación". Setiembre 2003

4. Comisión Interamericana Contra el Abuso de Drogas de la Organización de Estados Americanos (CICAD/OEA)- Oficina Panamericana de la Salud (OPS/OMS) (2000) "Guía Práctica para la organización de un Sistema Integral de Tratamiento de la Dependencia de Drogas". 2004

5. Larban Vera J (2008). "El Modelo Comunitario de Atención de Atención a la Salud Mental: Continente y Contenido". CUADERNOS DE PSIQUIATRÍA Y PSICOTERAPIA DEL NIÑOY DEL ADOLESCENTE, 2010; 49, 205-224

6. López Austin, A. (Coordinador). (2005) “El Modelo en la Ciencia y la Cultura”. Editorial Siglo XXI. Universidad Nacional Autónoma de México. México

7. Earls, J. (2011). "Introducción a la Teoría de Sistemas Complejos". Fondo Editorial de la Pontificia Universidad Católica del Perú. Lima-Perú.

8. Frenck, J. et al. “La transición epidemiológica en América Latina”. Instituto Nacional de Salud Pública de México. 1989

9. Gobierno de Canadá (1981). Informe Lalonde: “Una nueva perspectiva sobre la salud de los canadienses: documento de trabajo" Marc Lalonde. Ottawa, Canadá

10. Aaron Hogue, et al. Treatment Adherence, Competence, and Outcome in Individual and Family Therapy for Adolescent Behavior Problems. J Consult Clin Psychol. 2008 August; 76(4):

11. Nizama V. Innovación Conceptual en Adicciones. Rev. De NeuroPsiquiatría. Rev. Neuropsiquiatra 78(1), 2015.
12. Oficina de Naciones Unidas para las Drogas y el Delito (UNODD) (2003)) "Tratamiento Contemporáneo del Abuso de Drogas: Análisis de las Pruebas Científicas". Nueva York. USA.

13. Oficina de Naciones Unidas para las Drogas y el Delito (ONUDD) (2003). Manual sobre Tratamiento del Abuso de Drogas. Abuso de drogas: Tratamiento y Rehabilitación. Guía práctica de planificación y aplicación. Nueva York 2003

14. Jacoby, E. “Viejos Problemas y nuevas epidemias: el reto de la transición en salud en el Perú". En Salud, Equidad y Pobreza en el Perú: Teoría y nuevas Tendencias. Centro de Investigación de la Universidad del Pacífico. Lima, 2002

15. Cynthia L. Rowe, Ph.D. Multidimensional Family Therapy: Addressing CoOccurring Substance Abuse and Other Problems among Adolescents with Comprehensive Family-Based Treatment. Child Adolesc Psychiatr Clin N Am. 2010 July; 19(3): 563-576.

16. Couwenbergh C, van den Brink W, Zwart K, et al. Comorbid psychopathology in adolescents and young adults treated for substance use disorders. Eur Child Adolesc Psychiatry 2006;15:319-328. [PubMed: 16648966]

17. Hawkins EH. A tale of two systems: Co-Occurring mental health and substance abuse disorders treatment for adolescents. Annu Rev Psychol 2009;60:197-227. [PubMed: 19035824]

18. Kaminer Y, Connor DF, Curry JF. Treatment of comorbid adolescent cannabis use and major depressive disorder. Psychiatry 2008:9. [PubMed: 18284670]

19. Kandel DB, Huang FY, Davies M. Comorbidity between patterns of substance use dependence and psychiatric syndromes. Drug Alcohol Depend 2001;64(2):233-41. [PubMed: 11543993]

20. McElheny, V. (2012).“Drawing the Map of Life:Inside the Human Genome Project". A Merloyd Lawrence Book. New York 
21. Armstrong TD, Costello EJ. Community studies on adolescent substance use, abuse, or dependence and psychiatric comorbidity. J Consult Clin Psychology 2002;70(6):1224-39.

22. Chan YF, Dennis ML, Funk RR. Prevalence and comorbidity of major internalizing and externalizing problems among adolescents and adults presenting to substance abuse treatment. J Subst Abuse Treat 2008;34:1424. [PubMed: 17574804]

23. Robbins MS, Kumar S, Walker-Barnes C, et al. Ethnic differences in comorbidity among substanceabusing adolescents referred to outpatient therapy. J Am Acad Child Adolesc Psychiatry 2002;41(4):394-401. [PubMed: 11931595]

24. Rowe $\mathrm{CL}$, Liddle $\mathrm{HA}$, Greenbaum $\mathrm{PE}$, et al. Impact of psychiatric comorbidity on treatment of adolescent drug abusers. Journal Subst Abuse Treat 2004;26(2):129-40.

25. Vreugdenhil C, Van Den Brink W, Wouters LF, et al. Substance use, substance used disorders, and comorbidity patterns in a representative sample of incarcerated male Dutch adolescents. J Nerv Ment Dis 2003;191:372-8. [PubMed: 12826918]

26. Cornelius JR, Maisto SA, Pollock NK, et al. Rapid relapse generally follows treatment for substance use disorders among adolescents. Addict Behav 2003;28(2):381-6. [PubMed:12573689]

27. Grella CE, Hser Yl, Joshi V, et al. Drug treatment outcomes for adolescents with comorbid mental health and substance abuse disorders. J Nerv Ment Dis 2001;189(6):384-92. [PubMed: 11434639]

28. Riggs, PDG.; Whitmore, EJ. Disruptive behavior disorders in children and adolescent. American Psychiatric Press; Washington DC: 1999. Substance use disorders and disruptive behavior disorders.

29. Clingempeel WG, Britt SC, Henggeler SW. Beyond treatment effects: Comorbid psychopathologies and long term outcomes among substanceabusing delinquents. Am J

Orthopsychiatry 2008;78(1):29-36. [PubMed: 18444724]

30. Myers MG, Stewart DG, Brown SA. Progression from conduct disorder to antisocial personality disorder following treatment for adolescent substance abuse. Am J Psychiatry 1998;155:479-485. [PubMed: 9545992]

31. Jessor, R.; Jessor, SL. Problem behavior and psychosocial development: a longitudinal study of youth. Academic Press; New York: 1977.

32. D'Amico EJ, Edelen MO, Miles JNV, et al. The longitudinal association between substance use and delinquency among high-risk youth. Drug Alcohol Depend 2008;93:85-92. [PubMed: 17977669]
33. Reinherz HG, Giaconia RM, Hauf AM, et al. General and specific childhood risk factors for depression and drug disorders by early adulthood. J Am Acad Child Adolesc Psychiatry 2000;39(2):223-31. [PubMed: 10673834]

34. Zucker RA, Donovan JE, Masten AS, et al. Early developmental processes and the continuity of risk for underage drinking and problem drinking. Pediatrics 2008;121:S252-72. [PubMed: 18381493]

35. Glantz MD. Introduction to the special issue on the impact of child psychopathology interventions on subsequent substance abuse: Pieces of the puzzle. J Consult Clin Psychology 2002;70(6):1203-06.

36. Spooner C, Hall W. Public policy and the prevention of substance abuse disorders. Current Opinion in Psychiatry 2002;15:235-239.

37. Rowe CL, Liddle HA. Substance abuse. J Marital Fam Therapy 2003;29(1):97-120.

38. Brody GH, Beach SRH, Philibert RA, et al. Parenting moderates a genetic vulnerability factor in longitudinal increases in youths' substance use. J Consult Clin Psychology 2009;77(1):1-11.

39. Hogue A, Liddle HA. Family-based treatment for adolescent substance abuse: controlled trials and new horizons in services research. Journal of Family Therapy 2009;31:126-54.

40. Williams RJ, Chang SY. A comprehensive and comparative review of adolescent substance abuse and treatment outcome. Clinical Psychology: Science and Practice 2000:138-66.

41. AACAP. Practice parameters for the assessment and treatment of children and adolescents with substance use disorders. J Am Acad Child Adolesc Psychiatry 2005:44.

42. Shelly F. Greenfield, and cols. Gender Research in the National Institute on Drug Abuse National Treatment Clinical Trials Network: A Summary of Findings. Am J Drug Alcohol Abuse. 2011 Sep; 37(5): 301-312.

43. Pier Vincenzo Piazza \& Véronique Deroche-Gamonet. A multistep general theory of transition to addiction. Psychopharmacology (2013) 229:387-413.

44. George F Koob. and Nora D Volkow. Neurocircuitry of Addiction. Neuropsychopharmacology REVIEWS (2010) 35, 217-238

45. Jennifer L. Perry. Prefrontal Cortex and Drug Abuse Vulnerability: Translation to Prevention and Treatment Interventions. Brain Res Rev. 2011 January 1;65(2): 124-149.

46. Nizama V. Modelo holístico de las adicciones centrado en la familia. Fondo editorial de la asamblea Nacional de rectores. 2013.

47. Nizama V. Tratamiento Familiar Holístico de las Adicciones. Editorial de la UNMSM. 2009.

\section{Indizado en: latindex}

http://www.latindex.org/latindex/ficha?folio $=14280$

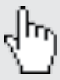

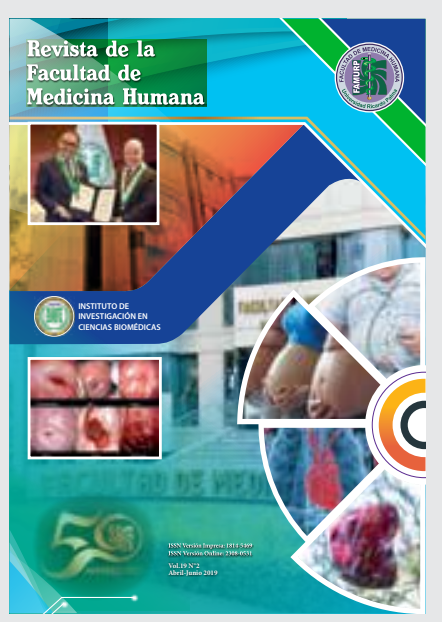

